\title{
Elevated CSF Neuron-specific Enolase Levels in Amyotrophic Lateral Sclerosis (Als): A Useful Biomarker for Distinguishing ALS From Cervical Spondylotic Myelopathy
}

\section{Akihiro Tsukahara}

Osaka Medical and Pharmaceutical University

Takafumi Hosokawa ( $\nabla$ takafumi.hosokawa@ompu.ac.jp )

Osaka Medical and Pharmaceutical University

Daisuke Nishioka

Osaka Medical and Pharmaceutical University

\section{Takuya Kotani}

Osaka Medical and Pharmaceutical University

\section{Shimon Ishida}

Osaka Medical and Pharmaceutical University

\section{Tohru Takeuchi}

Osaka Medical and Pharmaceutical University

\section{Fumiharu Kimura}

Osaka Medical and Pharmaceutical University Mishima- Minami Hospital

\section{Shigeki Arawaka}

Osaka Medical and Pharmaceutical University

\section{Research Article}

Keywords: Neuron-specific enolase, Amyotrophic lateral sclerosis, Cervical spondylotic myelopathy, Cerebrospinal fluid

Posted Date: August 18th, 2021

DOl: https://doi.org/10.21203/rs.3.rs-805919/v1

License: (1) This work is licensed under a Creative Commons Attribution 4.0 International License. Read Full License 


\section{Abstract}

The current study aimed to evaluate whether cerebrospinal fluid (CSF) neuron-specific enolase (NSE) levels are elevated in amyotrophic lateral sclerosis (ALS) and are effective in distinguishing ALS from cervical spondylotic myelopathy (CSM). We retrospectively evaluated 45 patients with ALS, 23 with CSM, and 28 controls who underwent analysis of CSF NSE levels. The control group comprised patients aged above 45 years who underwent lumbar puncture because of suspected neurological disorders that were ruled out after extensive investigations. CSF NSE levels were evaluated using the electrochemiluminescent immunoassay. The ALS group had significantly higher CSF NSE levels than the CSM and control groups ( $P<0.001$ for both comparisons). The CSM and control groups did not significantly differ in terms of CSF NSE levels. A receiver-operating characteristic curve analysis was performed to assess the diagnostic value of CSF NSE levels in distinguishing ALS from CSM. The area under the curve for CSF NSE levels was 0.86 . The optimal cutoff value was $17.7 \mathrm{ng} / \mathrm{mL}$, with a specificity of $87 \%$ and a sensitivity of $80 \%$. Hence, CSF NSE levels are elevated in ALS and are effective in distinguishing ALS from CSM.

\section{Introduction}

Amyotrophic lateral sclerosis (ALS) is a progressive and fatal disease characterized by the neurodegeneration of both upper and lower motor neurons. The pathogenesis of the condition is unclear, and its diagnosis is made clinically ${ }^{1}$. As there are no specific tests for ALS, a detailed set of diagnostic criteria has been established ${ }^{2}$. However, some patients with ALS patients do not fulfill the clinical criteria on ALS particularly at the early stage, and they are misdiagnosed with different neurological and medical disorders $^{1,3}$. Importantly, a misdiagnosis of cervical spondylotic myelopathy (CSM) is an important problem. ALS is most commonly misdiagnosed as $\mathrm{CSM}^{4,5}$. Patients with ALS present with focal muscle weakness and atrophy without bulbar symptoms at the early stage of the disease $\mathrm{e}^{3,6}$, which is similar to cervical spondylosis (CS). Among CS, ALS characterized by lower limb spasm but without radicular pain might be easy to distinguish from cervical spondylotic radiculopathy, but not from CSM. Further, a misdiagnosis of CSM may lead to unnecessary surgery 7,8 and subsequently more rapid deterioration because some patients with ALS experience accelerated disease progression after operation ${ }^{9,10}$. Spinal magnetic resonance imaging (MRI) is a useful but not a sufficient tool for distinguishing ALS from CSM because patients frequently experience concomitant $\mathrm{CS}^{11}$. Therefore, a novel tool is required to differentiate ALS from CSM.

Neuron-specific enolase (NSE) is a glycolytic enzyme predominantly observed in neurons and endocrine cells ${ }^{12}$. The intraneuronal NSE is secreted into the extracellular space after substantial neuronal damage. However, NSE is not physically secreted. Therefore, an elevated CSF NSE level mainly reflects neuronal damage $^{13}$. In fact, this phenomenon is observed in different conditions associated with central nervous system damage, such as traumatic brain injury ${ }^{14}$, traumatic spinal cord injury ${ }^{15}$, acute brain infarction ${ }^{16,17}$, Alzheimer's disease ${ }^{18}$ multiple system atrophy ${ }^{19}$, bacterial meningoencephalitis ${ }^{20}$, and 
Creutzfeldt-Jakob disease ${ }^{21}$. However, thus far, there have been no reports, at least those written in English, about CSF NSE levels in ALS.

Therefore, this study investigated whether CSF NSE levels are elevated in ALS and whether they are a useful biomarker for distinguishing ALS from CSM.

\section{Materials And Methods}

\section{Patients}

We retrospectively evaluated 45 patients with ALS, 23 with CSM, and 28 controls who were admitted to Osaka Medical and Pharmaceutical University Hospital and who underwent lumbar puncture and subsequent analysis of CSF NSE levels from January 2014 to January 2021. Patients were diagnosed with definite, probable, or probable laboratory-supported (PLS) ALS according to the revised El Escorial criteria $^{2}$. Those with ALS and concomitant CSM were classified under the ALS group. CSM was diagnosed based on the presence of myelopathic symptoms, such as limb numbness, problems with fine motor skills, and gait disturbance, and radiologic cervical cord compression in the stenotic canal, which is correlated with the patients' symptoms. The control group comprised patients aged above 45 years who underwent lumbar puncture due to suspected neurological disorders that were ruled out after extensive investigations. Not only controls and patients diagnosed with ALS and CSM at the time of CSF sampling but also those diagnosed at a later time (up to February 2021) were included. Patients with other concomitant neurological or neuromuscular disorders were excluded.

Information about age, sex, disease duration, neurological symptoms, disability, spinal MRI findings, and ALS categories according to the El Escorial criteria were collected at the time of CSF sampling. Based on the presence of a concave defect in the cervical cord caused by the impingement of the disc or osseous material on MRI regardless of defect degree and its symptoms, cervical cord compression was considered. Disability associated with ALS was determined using the Revised ALS Functional Rating Scale Score (ALSFRS-R), which has a maximum of 48 points. Lower scores represent a more severe disease stage $^{28}$.

This study was conducted according to the 2013 Helsinki Declaration, and the Osaka Medical and Pharmaceutical University Ethics Committee approved the study protocol and the need for informed consent was waived because this was a retrospective study and the data were collected without individual patient identifiers (Approval number \# 2020-189).

\section{CSF NSE analysis}

CSF samples were collected via lumbar puncture. Then, they were immediately brought to the laboratory for analysis. CSF NSE levels were evaluated using the electro-chemiluminescent immunoassay performed by SRL (Tokyo, Japan). The detection limit was $0.1 \mathrm{ng} / \mathrm{mL}$. 


\section{Statistical analysis}

The Mann-Whitney $U$ test was used to assess differences in continuous variables between two groups. The Kruskal-Wallis test, followed by the Dunn's multiple comparison test, was utilized to evaluate differences between three groups. Meanwhile, the chi-square test was applied to examine categorical variables. To investigate the accuracy of biomarkers in differentiating ALS from CSM, a receiver operating characteristic (ROC) curve analysis was performed by calculating the area under the ROC curve (AUC). The optimal cutoff value was chosen using the maximized Youden index. The values were expressed as mean \pm standard deviation, and a $P$ value of $<0.05$ was considered statistically significant. All analyses were performed using the JMP software version 15.0 (SAS Institute Inc., Cary, NC, the USA).

\section{Results}

\section{Characteristics of patients}

The characteristics of the three groups at time of CSF sampling are shown in Table 1. One-third of patients with ALS did not present with bulbar symptoms, and about one-half had cervical cord compression on MRI. All patients finally fulfilled the criteria on definite, probably, or PLS ALS. However, approximately one-half did not meet the criteria at time of CSF sampling. There were no significant differences in terms of age between the ALS, CSM, and control groups. However, the proportion of male patients between the three groups remarkably differed $(P=0.021)$. Nevertheless, there was no significant difference in terms of disease duration between the ALS and CSM groups. All patients with CSM had cervical cord compression on MRI according to the inclusion criteria of this study. Further, there were significant differences in the proportion of patients with cervical cord compression between the three groups $(P<0.001)$.

\section{CSF NSE levels}

The ALS group (mean \pm standard deviation: $21.0 \pm 5.1 \mathrm{ng} / \mathrm{mL}$ ) had significantly higher CSF NSE levels than the CSM $(13.7 \pm 4.3 \mathrm{ng} / \mathrm{mL}, P<0.001)$ and control $(13.6 \pm 4.0 \mathrm{ng} / \mathrm{mL}, P<0.001)$ groups (Fig. 1$)$. There was no significant difference in terms of CSF NSE levels between the CSM and control groups. To control the confounding effects of age and sex, we further performed several subgroup analyses of male and female patients and those aged $<70$ and $\geq 70$ years, respectively (Fig. 2). In the subgroup analyses of men and those aged $<70$ and $\geq 70$ years, the ALS group had significantly higher CSF NSE levels than the CSM group ( $P<0.001$ for male patients, $P=0.001$ for those aged $<70$ years, and $P=0.002$ for those aged $\geq 70$ years) and the control group ( $P<0.001$ for male patients, $P=0.017$ for those aged $<70$ years, and $P<0.001$ for those aged $\geq 70$ years). The CSF NSE levels did not differ between the CSM and control groups. In a subgroup analysis of women, the ALS group had significantly higher CSF NSE levels than the control group $(P=0.001)$. Moreover, the ALS group had higher CSF NSE levels than the CSM group ( $P=$ 0.133 ) although the results did not significantly differ possibly due to the small sample size. An ROC curve analysis was performed to assess the diagnostic value of CSF NSE levels in distinguishing ALS 
from CSM (Fig. 3). The AUC of CSF NSE levels was 0.86 . The optimal cutoff value was $17.7 \mathrm{ng} / \mathrm{mL}$, with a specificity of $87 \%$ and sensitivity of $80 \%$.

Figure 4 shows the associations between CSF NSE levels and clinical characteristics in patients with ALS at the time of CSF sampling. Patients with possible or suspected ALS $(22.5 \pm 5.2 \mathrm{ng} / \mathrm{mL})$ had significantly higher CSF NSE levels than those with definite, probable, or PLS ALS $(19.5 \pm 4.5 \mathrm{ng} / \mathrm{mL}, P=$ 0.046). Moreover, the CSF NSE levels were significantly higher in patients with ALS who had an ALSFRS-R score of $>36(22.4 \pm 5.3 \mathrm{ng} / \mathrm{mL})$ than in those with an ALSFRS-R score of $\leq 36(19.2 \pm 4.3 \mathrm{ng} / \mathrm{mL}, P=$ 0.037). There were no significant differences in terms of CSF NSE levels between patients with and without bulbar symptoms; those who had a disease duration of $\leq 12$ and $>12$ months; and those with and without cervical cord compression on MRI. ROC curve analysis was performed to assess the diagnostic value of CSF NSE levels in distinguishing ALS with several features from CSM. The AUCs of CSF NSE levels were 0.91 in ALS without bulbar symptoms, 0.87 in ALS with cervical cord compression, and 0.91 in ALS that do not fulfill the criteria on definite, probable, or PLS.

\section{Discussion}

The primary finding of this study is that CSF NSE levels are elevated in ALS. Based on a previous study, they are influenced by age and sex ${ }^{22}$. Moreover, this research showed elevated CSF NSE levels in ALS via the subgroup analyses of male and female patients and those aged $<70$ and $\geq 70$ years, which were performed to control the confounding effects of age and sex. To the best of our knowledge, this study, at least among those written in English, first showed elevated CSF NSE levels in ALS. Another main finding is that CSF NSE levels are higher in ALS than in CSM; therefore, they are useful in distinguishing ALS from CSM. In relation to the finding, the CSF NSE levels were not elevated in CSM. In the literature, the CSF NSE levels in CSM are controversial. That is, a previous report showed high CSF NSE levels in $\mathrm{CSM}^{23}$. Meanwhile, another revealed normal levels ${ }^{24}$. Taken together, CSF NSE levels in CSM may not be as elevated as those in ALS. Hence, they can be used to distinguish ALS from CSM.

There are several explanations why the ALS group had higher CSF NSE levels than not only the control but also CSM groups, even though NSE is generally a non-specific marker of neural damage ${ }^{13}$. First, the difference in CSF NSE levels may reflect different degrees of neural damage. A widespread and aggressive neural damage in ALS can result in significantly elevated CSF NSE levels. However, a limited and non-aggressive neural damage in CSM may not. Second, differences in CSF NSE levels can reflect varying affected areas. For example, the grey matter, which has high NSE levels, could be involved in ALS but not in CSM $^{25}$. Third, differences in CSF NSE levels may reflect varying pathologic processes. That is, NSE may not be a non-specific marker of neural damage. However, it may play a role in several pathologic processes, and the mechanism might occur in ALS. In fact, NSE has been involved in pathologic processes such as neuroinflammation, particularly in the expression of pro-inflammatory cytokines and the proliferation of inflammatory glial cells ${ }^{25,26}$. In addition, the importance of 
neuroinflammation in ALS has been reported ${ }^{27}$. However, specific pathologic processes leading to elevated CSF NSE levels in ALS is not addressed.

Distinguishing ALS from CSM is challenging, particularly when patients with ALS present with cervical cord compression on MRI and they do not experience bulbar symptoms and do not fulfill the criteria on ALS. In this study, patients with ALS and such features had significantly higher CSF NSE levels than those with ALS without such features, or the CSF NSE levels of the former group was as high as those of the latter group. In detail, patients with ALS who do not fulfil the criteria on definite, probable, or PLS ALS had significantly higher CSF NSE levels than those who fulfilled the criteria. The CSF NSE levels of patients with ALS with cervical cord compression was as high as those of patients with ALS without compression. Moreover, the CSF NSE levels of patients with ALS without bulbar symptoms was as high as those of patients with ALS with the symptoms. Consequently, the diagnostic values of CSF NSE levels in distinguishing ALS with such features from CSM were higher or as high as those of CSF NSE levels in distinguishing whole ALS from CSM. In cases in which patients with ALS are challenging to distinguish from those with CSM, CSF NSE can be used. Hence, it may be an effective biomarker.

The reason why patients with ALS who do not fulfil the criteria had significantly higher CSF NSE levels than those who fulfilled the criteria is uncertain. However, it could be explained by a hypothesis that CSF NSE levels might decrease with disease progression at a certain stage because it could be accompanied by a decreased number of motor neurons, which might be the source of CSF NSE. Notably, the hypothesis could also explain our findings that patients with mild ALS had higher CSF NSE levels than other patients.

Our study had several limitations. First, it had a small sample size and was retrospective in nature. Hence, further large prospective studies should be conducted. Second, the control group only comprised unhealthy patients who underwent lumbar puncture, which is an invasive test, because of suspected neurological disorders that were ruled out after extensive investigations.

CSF NSE levels are elevated in ALS. Further, they can effectively distinguish ALS from CSM and prevent the misdiagnosis of CSM in patients with ALS. Thus, unnecessary surgery and subsequent rapid deterioration may be prevented. Notably, numerous physicians including those in general medical institutions can benefit from the use of this biomarker in daily clinical practice because NSE is a common tumor marker for diseases including small lung cancer and can be measured in general medical institutions. In addition, because elevated CSF NSE levels in ALS may reflect a specific pathologic process, this finding could provide new perspectives regarding the understanding of ALS pathogenesis and could facilitate the development of appropriate treatments.

\section{Declarations}

\section{Author contributions}


A.T. designed the study, carried out the acquisition of data, and wrote the manuscript. T.H. designed the study, carried out the acquisition of data, analyzed the data, and wrote the manuscript. D.N. analyzed the data. T.K. supervised the manuscript. S.I. supervised the manuscript. T.T. supervised the manuscript. F.K. supervised the manuscript. S.A. revised the manuscript. All authors reviewed the manuscript.

\section{Competing Interests}

The authors declare no competing interests.

\section{Additional information}

Correspondence and requests for materials should be addressed to T.H.

\section{References}

1. Hardiman, O., van den Berg, L. H. \& Kiernan, M. C. Clinical diagnosis and management of amyotrophic lateral sclerosis. Nat Rev Neurol, 7, 639-649 https://doi.org/10.1038/nrneurol.2011.153 (2011).

2. Brooks, B. R., Miller, R. G., Swash, M., Munsat, T. L. \& World Federation of Neurology Research Group on Motor. Neuron, D. El Escorial revisited: revised criteria for the diagnosis of amyotrophic lateral sclerosis. Amyotroph Lateral Scler Other Motor Neuron Disord, 1, 293-299 https://doi.org/10.1080/146608200300079536 (2000).

3. Jacobson, R. D., Goutman, S. A. \& Callaghan, B. C. Pearls \& Oy-sters: The importance of atypical features and tracking progression in patients misdiagnosed with ALS. Neurology, 86, e136-139 https://doi.org/10.1212/WNL.0000000000002522 (2016).

4. Cellura, E., Spataro, R. \& Taiello, A. C. \& La Bella, V. Factors affecting the diagnostic delay in amyotrophic lateral sclerosis. Clin Neurol Neurosurg, 114, 550-554 https://doi.org/10.1016/j.clineuro.2011.11.026 (2012).

5. Rowland, L. P. Diagnosis of amyotrophic lateral sclerosis. J Neurol Sci, 160 Suppl (1), S6-24 https://doi.org/10.1016/s0022-510x(98)00193-2 (1998).

6. Williams, J. R., Fitzhenry, D., Grant, L., Martyn, D. \& Kerr, D. A. Diagnosis pathway for patients with amyotrophic lateral sclerosis: retrospective analysis of the US Medicare longitudinal claims database. BMC Neurol, 13, 160 https://doi.org/10.1186/1471-2377-13-160 (2013).

7. Kraemer, M., Buerger, M. \& Berlit, P. Diagnostic problems and delay of diagnosis in amyotrophic lateral sclerosis. Clin Neurol Neurosurg, 112, 103-105 https://doi.org/10.1016/j.clineuro.2009.10.014 (2010).

8. Yoshor, D., Klugh, A. 3rd, Appel, S. H. \& Haverkamp, L. J. Incidence and characteristics of spinal decompression surgery after the onset of symptoms of amyotrophic lateral sclerosis. Neurosurgery 57, 984-989; discussion 984-989, doi:10.1227/01.neu.0000180028.64385.d3 (2005). 
9. Sostarko, M., Vranjes, D., Brinar, V. \& Brzovic, Z. Severe progression of ALS/MND after intervertebral discectomy. J Neurol Sci, 160 Suppl (1), S42-46 https://doi.org/10.1016/s0022-510x(98)00197-x (1998).

10. Pinto, S., Swash, M. \& de Carvalho, M. Does surgery accelerate progression of amyotrophic lateral sclerosis? J Neurol Neurosurg Psychiatry, 85, 643-646 https://doi.org/10.1136/jnnp-2013-305770 (2014).

11. Yamada, M., Furukawa, Y. \& Hirohata, M. Amyotrophic lateral sclerosis: frequent complications by cervical spondylosis. J Orthop Sci, 8, 878-881 https://doi.org/10.1007/s00776-003-0712-0 (2003).

12. Kato, K., Ishiguro, Y., Suzuki, F., Ito, A. \& Semba, R. Distribution of nervous system-specific forms of enolase in peripheral tissues. Brain Res, 237, 441-448 https://doi.org/10.1016/00068993(82)90455-3 (1982).

13. Isgro, M. A., Bottoni, P. \& Scatena, R. Neuron-Specific Enolase as a Biomarker: Biochemical and Clinical Aspects. Adv Exp Med Biol, 867, 125-143 https://doi.org/10.1007/978-94-017-7215-0_9 (2015).

14. Herrmann, M. et al. Protein S-100B and neuron specific enolase as early neurobiochemical markers of the severity of traumatic brain injury. Restor Neurol Neurosci, 14, 109-114 (1999).

15. Pouw, M. H. et al. Structural biomarkers in the cerebrospinal fluid within $24 \mathrm{~h}$ after a traumatic spinal cord injury: a descriptive analysis of 16 subjects., 52, 428-433 https://doi.org/10.1038/sc.2014.26 (2014).

16. Selakovic, V., Raicevic, R. \& Radenovic, L. The increase of neuron-specific enolase in cerebrospinal fluid and plasma as a marker of neuronal damage in patients with acute brain infarction. J Clin Neurosci, 12, 542-547 https://doi.org/10.1016/j.jocn.2004.07.019 (2005).

17. Hay, E. et al. Cerebrospinal fluid enolase in stroke. J Neurol Neurosurg Psychiatry, 47, 724-729 https://doi.org/10.1136/jnnp.47.7.724 (1984).

18. Christl, J. et al. Association of Cerebrospinal Fluid S100B Protein with Core Biomarkers and Cognitive Deficits in Prodromal and Mild Alzheimer's Disease. J Alzheimers Dis, 72, 1119-1127 https://doi.org/10.3233/JAD-190550 (2019).

19. Abdo, W. F. et al. Cerebrospinal fluid analysis differentiates multiple system atrophy from Parkinson's disease. Mov Disord, 19, 571-579 https://doi.org/10.1002/mds.10714 (2004).

20. Kepa, L. Evaluation of cerebrospinal fluid and plasma neuron-specific enolase (NSE) concentration in patients with purulent, bacterial meningoencephalitis. Przegl Epidemiol, 63, 23-28 (2009).

21. Zerr, I. et al. Cerebrospinal fluid concentration of neuron-specific enolase in diagnosis of CreutzfeldtJakob disease., 345, 1609-1610 https://doi.org/10.1016/s0140-6736(95)90118-3 (1995).

22. Nygaard, O., Langbakk, B. \& Romner, B. Neuron-specific enolase concentrations in serum and cerebrospinal fluid in patients with no previous history of neurological disorder. Scand J Clin Lab Invest, 58, 183-186 https://doi.org/10.1080/00365519850186562 (1998).

23. Yasuda, T., Yanagi, T., Mokuno, K. \& Kato, K. Neuron-specific enolase (NSE) and S-100b protein in cerebrospinal fluid of patients with cervical spondylosis-the relations with MRI findings and the 
changes of NSE and S-100b protein levels through Glisson's traction. Rinsho Shinkeigaku, 32, 12991301 (1992).

24. Takahashi, H. et al. Axonal damage is remarkable in patients with acutely worsening symptoms of compression myelopathy: biomarkers in cerebrospinal fluid samples. Eur Spine J, 27, 1824-1830 https://doi.org/10.1007/s00586-018-5549-5 (2018).

25. Haque, A., Polcyn, R., Matzelle, D. \& Banik, N. L. New Insights into the Role of Neuron-Specific Enolase in Neuro-Inflammation, Neurodegeneration, and Neuroprotection. Brain Sci, 8, https://doi.org/10.3390/brainsci8020033 (2018).

26. Haque, A., Ray, S. K., Cox, A. \& Banik, N. L. Neuron specific enolase: a promising therapeutic target in acute spinal cord injury. Metab Brain Dis, 31, 487-495 https://doi.org/10.1007/s11011-016-9801-6 (2016).

27. Chiot, A., Lobsiger, C. S. \& Boillee, S. New insights on the disease contribution of neuroinflammation in amyotrophic lateral sclerosis. Curr Opin Neurol, 32, 764-770 https://doi.org/10.1097/WC0.0000000000000729 (2019).

28. Cedarbaum, J. M. et al. The ALSFRS-R: a revised ALS functional rating scale that incorporates assessments of respiratory function. BDNF ALS Study Group (Phase III). J Neurol Sci, 169, 13-21 https://doi.org/10.1016/s0022-510x(99)00210-5 (1999).

\section{Tables}

Table 1: Characteristics of the ALS, CSM, and control groups at the time of CSF sampling 


\begin{tabular}{|lllll|}
\hline & $\begin{array}{l}\text { ALS group } \\
(\mathrm{n}=45)\end{array}$ & $\begin{array}{l}\text { CSM group } \\
(\mathrm{n}=23)\end{array}$ & $\begin{array}{l}\text { Control group } \\
(\mathrm{n}=28)\end{array}$ & Pvalue \\
\hline Age (years), mean \pm SD & $70.2 \pm 8.5$ & $67.4 \pm 10.0$ & $67.0 \pm 14.5$ & NS \\
\hline Male sex, $\mathrm{n}(\%)$ & $21(47)$ & $18(78)$ & $12(43)$ & 0.021 \\
\hline Disease duration (months), mean \pm SD & $14.8 \pm 11.1$ & $14.8 \pm 14.4$ & NA & NS \\
\hline Bulbar symptoms, $\mathrm{n}(\%)$ & $30(67)$ & NA & NA & \\
\hline ALSFRS-R, mean \pm SD & $36.1 \pm 8.2$ & NA & NA & \\
\hline Cervical cord compression on MRI, $\mathrm{n}(\%)$ & $21 / 41(51)$ & $23 / 23(100)$ & $7 / 21(33)$ & $<0.001$ \\
\hline El Escorial category & & & & \\
\hline Definite, $\mathrm{n}(\%)$ & $7(16)$ & NA & NA & \\
\hline Probable, $\mathrm{n}(\%)$ & $9(20)$ & NA & NA & \\
\hline PLS, $\mathrm{n}(\%)$ & $7(16)$ & NA & NA & \\
\hline Possible, $n(\%)$ & $13(29)$ & NA & NA & NA \\
\hline Suspected, $n(\%)$ & $9(20)$ & NA & & \\
\hline
\end{tabular}

Abbreviations: ALS, Amyotrophic lateral sclerosis; ALSFRS-R, Revised ALS Functional Rating Scale; CSF, cerebrospinal fluid; CSM, Cervical spondylotic myelopathy; NA, Not applicable; NS, Not significant; PLS, Probable laboratory-supported.

\section{Figures}




\section{$P<0.001$}

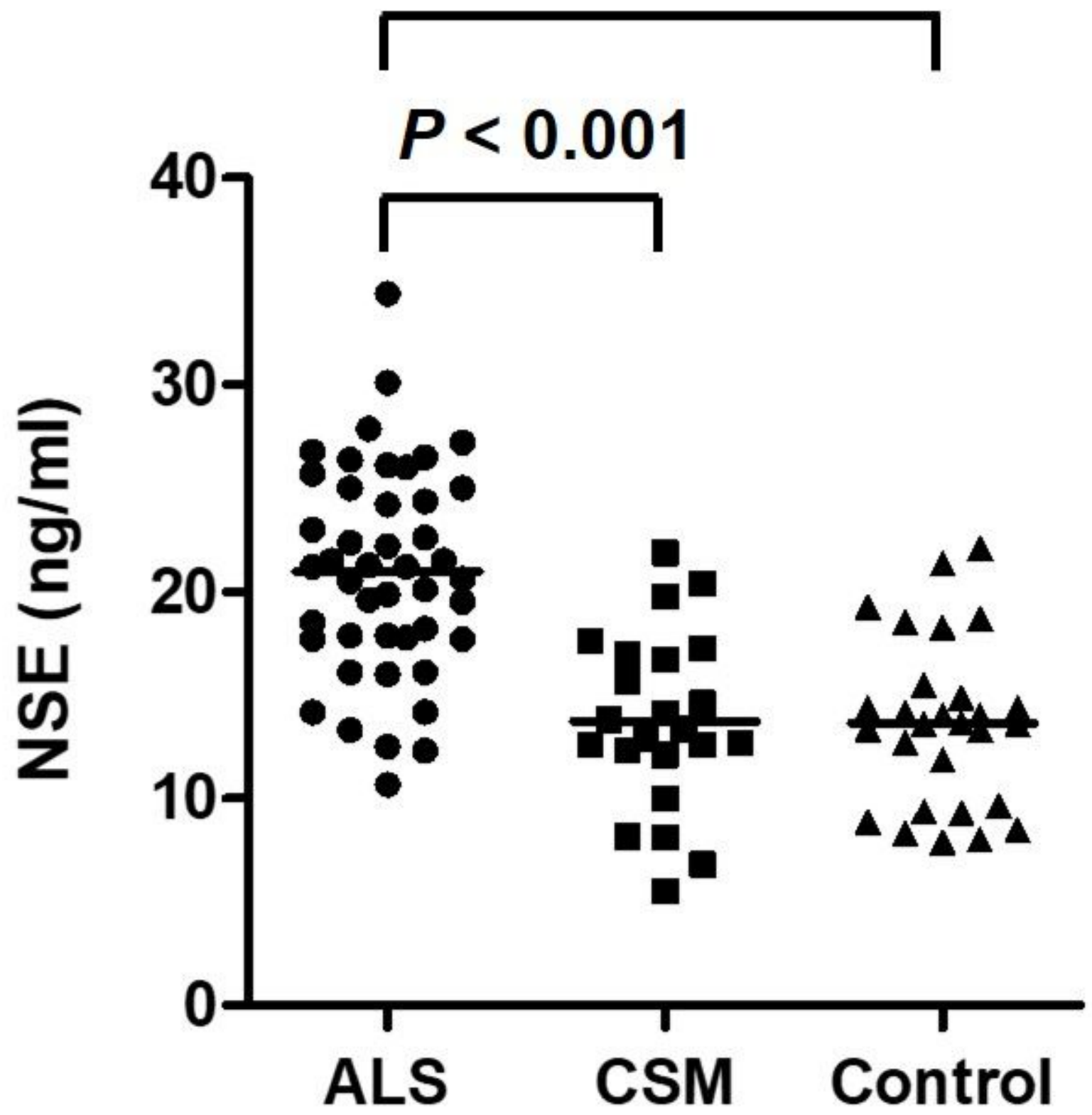

Figure 1

CSF NSE levels of the ALS, CSM, and control groups. The solid line represents the mean CSF NSE levels of each group. Abbreviation: ALS, amyotrophic lateral sclerosis; CSF, cerebrospinal fluid; CSM, cervical spondylotic myelopathy; NSE, neuron-specific enolase. 
A

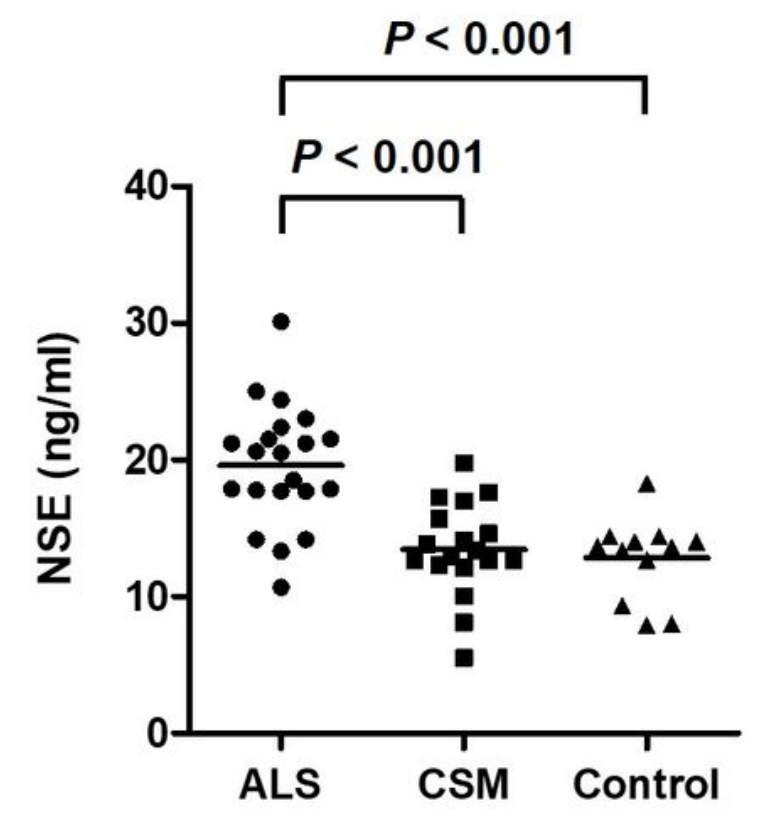

C Age $<70$ years

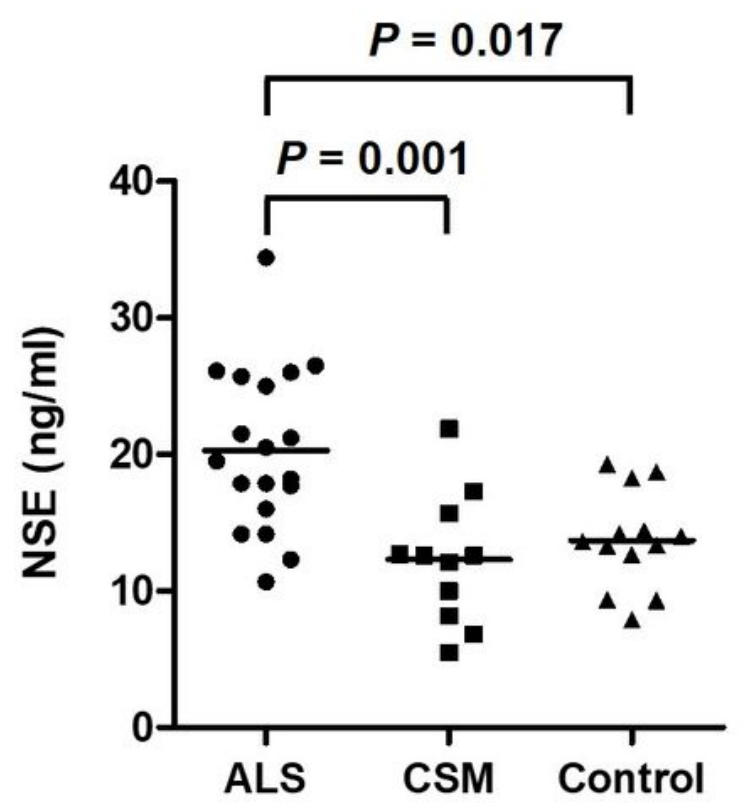

B

Female
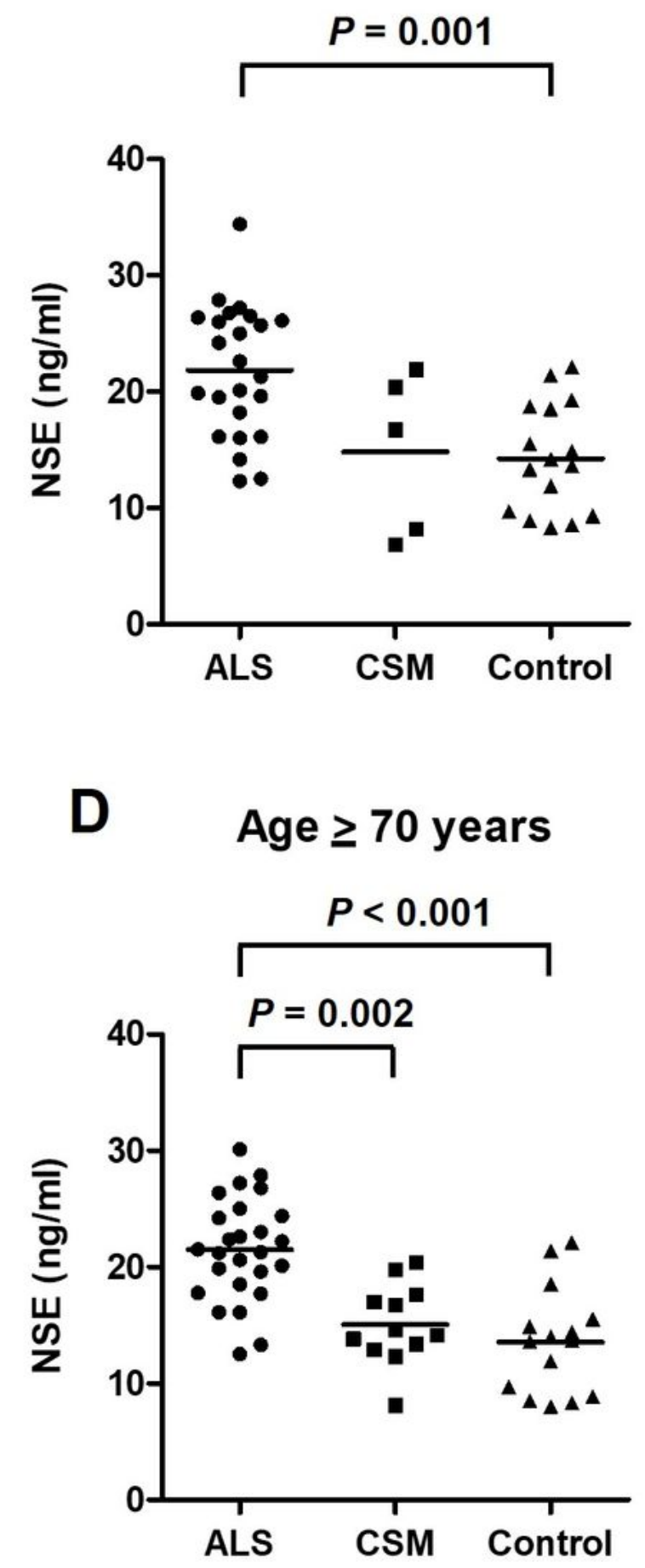

Figure 2

CSF NSE levels of the ALS, CSM, and control groups based on the subgroup analyses of (A) male and (B) female patients, (C) those aged $<70$ years, and (D) those aged $\geq 70$ years. The solid line represents the mean CSF NSE levels of each group. Abbreviation: ALS, amyotrophic lateral sclerosis; CSF, cerebrospinal fluid; CSM, cervical spondylotic myelopathy; NSE, neuron-specific enolase. 


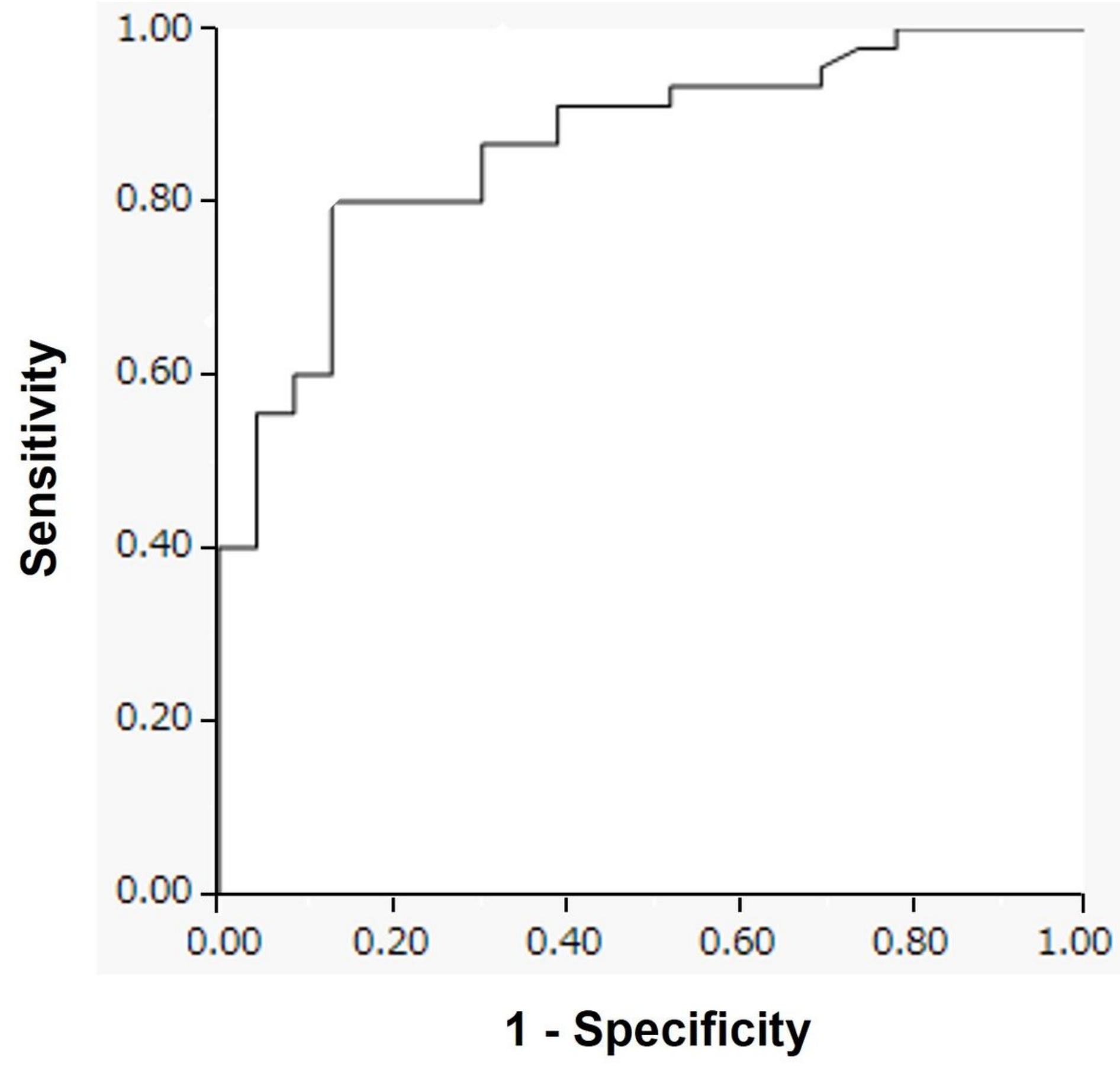

Figure 3

Receiver operating characteristic curves for distinguishing ALS from CSM based on CSF NSE levels. Abbreviation: ALS, amyotrophic lateral sclerosis; CSF, cerebrospinal fluid; CSM, cervical spondylotic myelopathy; NSE, neuron-specific enolase. 

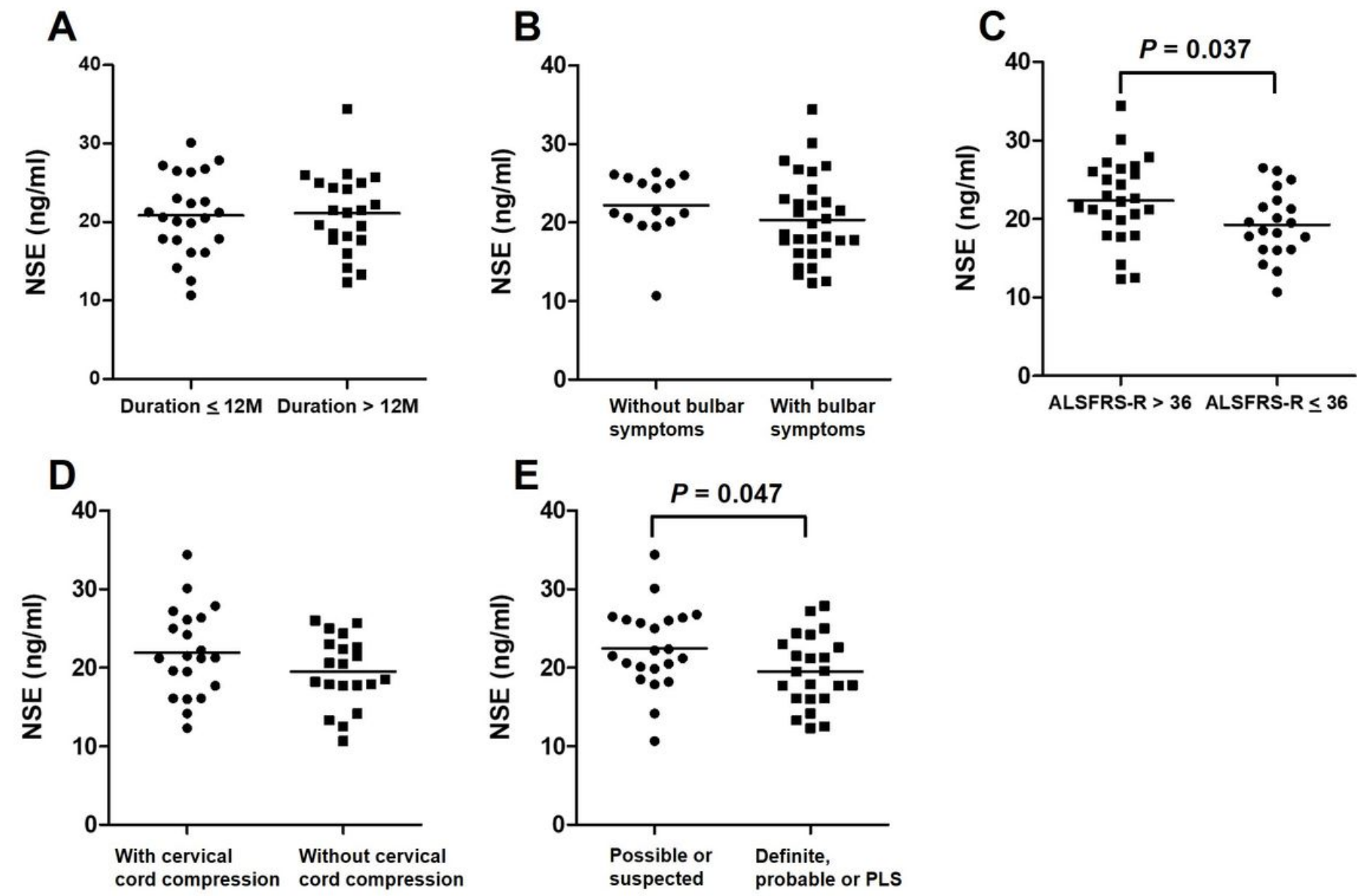

Figure 4

Associations between CSF NSE levels and clinical characteristics in patients with ALS at the time of CSF sampling. The CSF NSE levels of patients (A) with a disease duration of $\leq 12$ and $>12$ months, (B) those with and without bulbar symptoms, (C) those with an ALSFRS-R score of $>36$ and $\leq 36$, (D) those with and without cervical cord compression on MRI, and (E) those with possible or suspected ALS and definite, probable, or PLS ALS. The solid line represents the mean CSF NSE levels of each group. Abbreviation: ALS, amyotrophic lateral sclerosis; ALSFRS-R, Revised ALS Functional Rating Scale Score; CSF, cerebrospinal fluid; CSM, cervical spondylotic myelopathy; NSE, neuron-specific enolase; PLS, probable laboratory-supported. 